\title{
New directions for 2006
}

\author{
The Pharmacogenomics Journal (2006) 6, 1. \\ doi:10.1038/sj.tpj.6500367
}

The Pharmacogenomics Journal is now in its sixth year. Many changes are occurring in our field, which are reflected in the journal, both in terms of content and format.

We would like to note that the field of pharmacogenomics has evolved from the realm of promise to that of reality. The United States Food and Drug Administration (FDA) now examines pharmacogenetic information when considering approving drugs.

This year, the FDA approved BiDil $^{\circledR}$ (hydralazine and isosorbide dinitrate, NitroMed) as a drug for the treatment of heart failure in self-identified black patients. The FDA stated in a news release dated 23 June 2005 that this represented 'a step toward the promise of personalized medicine' (http:// www.fda.gov/bbs/topics/NEWS/2005/NEW01190.html).

'Today's approval of a drug to treat severe heart failure in self-identified black population is a striking example of how a treatment can benefit some patients even if it does not help all patients,' said Dr Robert Temple, FDA Associate Director of Medical Policy.

While race is a very poor surrogate for genetic background, the basic premise of pharmacogenomics, which is that pharmacologic treatment can be tailored to benefit specific groups, is now accepted official policy of the FDA. The challenge for the field then is to identify which groups will respond better to specific drugs.

An enormous advance in this search has been accomplished by completion of the HapMap project. ${ }^{1}$ This highly important new resource for pharmacogenomics consists of a public database of common variation in the human genome with more than one million single-nucleotide polymorphisms (SNPs) from four populations. The HapMap data document the generality of recombination hotspots, leading to substantial correlations of SNPs with many of their neighbors. This will guide the design and analysis of genetic association studies, including pharmacogenetic studies.

In a recent analysis of the future genomic medicine, Guttmacher and Collins stated that the broad impact of genomics on health in the near future should come from its applications to diagnostics, pharmacogenomics, and drug development'. Some genomic medicine has already started to move from promise to reality. An example in diagnostics is the use of multi-gene analysis to predict the need for chemotherapy in certain breast cancers. In pharmacogenomics, knowledge of a patient's genetic makeup can sometimes help avoid adverse drug reactions, as recent studies showed for warfarin. In drug development, genomics can already salvage a drug that might otherwise be abandoned, as demonstrated by gefitinib, which has little benefit for the majority of those with lung cancer, but may prove lifesaving in the sizable minority with a specific genotype. $^{2}$

Our editorial goal is to be the principal publication vehicle of substantial progress in the search for individualized medicine and new drug targets. We are delighted to inform our readers that this year we will have our first Impact Factor issued by the Institute for Scientific Information. In addition to original research that will define the field of pharmacogenomics in the years to come, we are calling for papers for the 'front of the book.' These include reviews, perspectives, meeting reports, clinical implications, and particularly EELS (ethical, economic, legal and social) articles. As the field advances, EELS issues become increasingly more relevant. We are the only journal with a specific section dedicated to this area, and therefore we count on our readers and authors to advance this important aspect of pharmacogenomics.

Thank you for the continued support given to The Pharmacogenomics Journal. In this sixth volume, we introduce a striking new cover and new formatting. We will of course continue to offer rigorous review, high visibility, and rapid publication.

J Licinio
Editor
Center for Pharmacogenomics \& Clinical Pharmacology, UCLA
Semel Institute and David Geffen School of Medicine,
Gonda Research Center 3357A, 695 Charles Young Drive So.,
Los Angeles, CA, USA
E-mail: licinio@ucla.edu

\section{REFERENCES}

1 Altshuler D, Brooks LD, Chakravarti A, Collins FS, Daly MJ, Donnelly P, International HapMap Consortium. A haplotype map of the human genome. Nature 2005; 437: 1299-1320.

2 Guttmacher AE, Collins FS. Realizing the promise of genomics in biomedical research. JAMA 2005; 294: 1399-1402. 